\title{
Mutations in the EGFR kinase domain mediate STAT3 activation via IL-6 production in human lung adenocarcinomas
}

\author{
Sizhi Paul Gao, ${ }^{1}$ Kevin G. Mark, ${ }^{1}$ Kenneth Leslie, ${ }^{1}$ William Pao, ${ }^{1}$ Noriko Motoi, ${ }^{2}$ \\ William L. Gerald, ${ }^{2}$ William D. Travis, ${ }^{2}$ William Bornmann, ${ }^{3}$ Darren Veach, ${ }^{1}$ \\ Bayard Clarkson, ${ }^{1}$ and Jacqueline F. Bromberg' \\ 1Department of Medicine and 2Department of Pathology, Memorial Sloan-Kettering Cancer Center (MSKCC), New York, New York, USA \\ ${ }^{3}$ Division of Experimental Diagnostic Imaging, University of Texas MD Anderson Cancer Center, Houston, Texas, USA.
}

\begin{abstract}
Persistently activated or tyrosine-phosphorylated STAT3 (pSTAT3) is found in 50\% of lung adenocarcinomas. pSTAT3 is found in primary adenocarcinomas and cell lines harboring somatic-activating mutations in the tyrosine kinase domain of EGFR. Treatment of cell lines with either an EGFR inhibitor or an src kinase inhibitor had no effect on PSTAT3 levels, whereas a pan-JAK inhibitor (P6) blocked activation of STAT3 and inhibited tumorigenesis. Cell lines expressing these persistently activated mutant EGFRs also produced high IL-6 levels, and blockade of the IL-6/gp130/JAK pathway led to a decrease in pSTAT3 levels. In addition, reduction of IL-6 levels by RNA interference led to a decrease in tumorigenesis. Introduction of persistently activated EGFR into immortalized breast epithelial cells led to tumorigenesis, IL-6 expression, and STAT3 activation, all of which could be inhibited with P6 or gP130 blockade. Furthermore, inhibition of EGFR activity in multiple cell lines partially blocked transcription of $I L-6$ and concurrently decreased production and release of IL-6. Finally, immunohistochemical analysis revealed a positive correlation between pSTAT3 and IL- 6 positivity in primary lung adenocarcinomas. Therefore, mutant EGFR could activate the gp130/JAK/STAT3 pathway by means of IL-6 upregulation in primary human lung adenocarcinomas, making this pathway a potential target for cancer treatment.
\end{abstract}

\section{Introduction}

Lung cancer is the leading cause of cancer deaths in the United States, resulting in approximately 160,000 deaths per year (1). Non-small cell lung cancer (NSCLC) accounts for more than $85 \%$ of all lung cancers. Adenocarcinomas, a major subtype of NSCLC, represent the most common histologic subtype of lung cancer in the United States and many countries worldwide. Some adenocarcinomas distinguish themselves by overexpressing wild-type EGFR and EGFR containing kinase domain-activating mutations (2-5).

EGFR is a membrane-bound receptor tyrosine kinase that belongs to the ErbB subfamily. On ligand binding, EGFRs initiate activation of a series of cellular signal transduction pathways that regulate cell proliferation and survival. The EGFR either is mutated or shows altered expression in a variety of human cancers, including carcinomas of the lung, breast, head and neck, ovary, and bladder as well as gliomas (6). Somatic-activating mutations in the tyrosine kinase domains of the EGFR are found in approximately $10 \%$ of lung adenocarcinomas $(3,4,7)$. Most lung adenocarcinoma-associated somatic EGFR tyrosine kinase domain mutations fall into 1 of 2 classes: in-frame deletions in exon 19

Nonstandard abbreviations used: CM, conditioned medium; CNTF, ciliary neurotropic factor; HNSCC, head and neck squamous cell carcinoma; LIF, leukemia inhibitory factor; NSCLC, non-small cell lung cancer; OSM, oncostatin M; P6, tetracyclic pyridone 2-tert-butyl-9-fluoro-3,6-dihydro-7H-benz[h]-imidaz[4,5-f]isoquinoline7-one; shRNA, short hairpin RNA; TKI, tyrosine kinase inhibitor; TMA, tissue microarray; TTF1, thyroid transcription factor 1 ; Tyk2, tyrosine kinase 2; ZD, ZD-1839 (gefitinib)

Conflict of interest: The authors have declared that no conflict of interest exists. Citation for this article: J. Clin. Invest. 117:3846-3856 (2007). doi:10.1172/JCI31871. that eliminate the conserved LREA motif ( $\triangle E$ EGFR) and a T-to-G base substitution in exon 21 that substitutes arginine for leucine at position 858 (L858R) (3). Patients whose tumors contain either of these 2 classes of mutations have increased sensitivity to the tyrosine kinase inhibitors (TKIs) gefitinib (ZD-1839 [ZD]) and erlotinib. In addition, lung cancer-derived cell lines harboring these kinase domain-activating mutant forms of the EGFR are sensitive to TKIs (8-10).

One of the important signaling mediators downstream of normally and abnormally activated EGFR is STAT3. STAT3 is a latent transcription factor found in the cytoplasm of cells. It is activated by tyrosine phosphorylation, leading to dimerization, nuclear translocation, DNA binding, and gene activation (11). In addition to EGFR, STAT3 can be activated by other receptor and nonreceptor tyrosine kinases, such as the IL-6/gp130 receptor family, PDGFR, src kinase, and JAKs. STAT3 is transiently activated in normal cells but constitutively activated in a wide variety of hematologic and epithelial primary tumors and tumor-derived cell lines, such as leukemias; lymphomas; head and neck, prostate, ovarian, breast, and renal cell cancers; and melanoma $(12,13)$. STAT3 is also persistently activated in about $50 \%$ of NSCLC primary tumors and lung cancer-derived cell lines (14-16). STAT3 is critically involved, primarily through differential gene regulation, with almost all aspects of tumorigenesis, including cell cycle progression, tumor invasion and metastasis, host immune system evasion, and tumor angiogenesis (17-19).

IL- 6 belongs to the IL- 6 cytokine family, which also contains oncostatin M (OSM), leukemia inhibitory factor (LIF), IL-11, and ciliary neurotropic factor (CNTF) (20). IL-6 regulates immune 


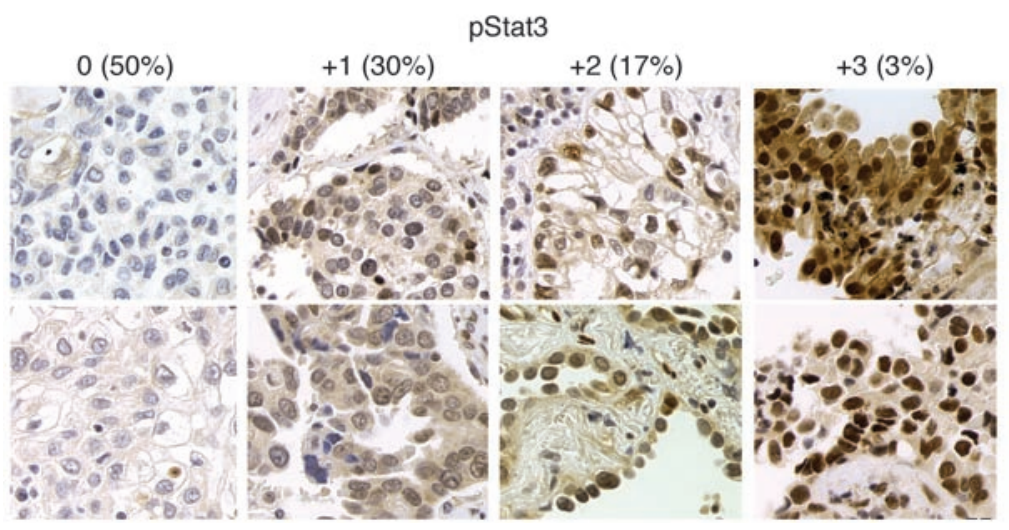

\section{Figure 1}

STAT3 is tyrosine phosphorylated in primary lung adenocarcinomas. Immunohistochemical analysis of 92 primary lung adenocarcinomas for tyrosine-phosphorylated STAT3 was performed; 46 were scored as $0 ; 27$ as +1 ; 16 as +2 ; and 3 as +3 , with associated percentages indicated in parentheses. Two examples of each are shown. Original magnification, $\times 200$. and inflammatory responses, but recent reports suggest that IL-6 expression is implicated in the regulation of tumor growth and metastatic spread, including lung cancers $(21,22)$. By combining with their ligand-specific binding subunit, IL- 6 and other IL-6 family cytokines use the common receptor gp130 as the signaling subunit to activate receptor-associated tyrosine kinases JAK1 and JAK2 as well as tyrosine kinase 2 (Tyk2) (20). JAK activation leads subsequently to phosphorylation of gp130 and recruitment and phosphorylation of signaling molecules, such as STAT3, MAPK, and PI3K $(13,21,23)$.

A positive correlation between elevated EGFR activity, including wild-type EGFR and kinase domain-activating mutant EGFR (referred to as mutant EGFR), and pSTAT3 has been described in many human primary tumor specimens and tumor-derived cell lines, including NSCLC as well as breast and head and neck tumors (9, 14-16, 24-27). Previous studies have demonstrated that TKI, src, or JAK2 inhibition of mutant EGFR-expressing cell lines did not lead to inhibition of STAT3 activation. Thus, the mechanisms of STAT3 phosphorylation and the phenotypic consequences of inhibiting STAT3 activity in either mutant EGFR-transfected cell lines or cancer-derived cell lines expressing mutant EGFR remain unclear $(8,14,28)$.

In this study, we discovered a strong correlation between PSTAT3 and adenocarcinomas harboring somatic-activating mutations in the tyrosine kinase domain of the EGFR ( $\triangle E$ EGFR and L858R). We determined that STAT3 activation in mutant EGFR-expressing cell lines occurs, in part, through mutant EGFR-mediated transcriptional upregulation of the IL- 6 gene, which, in turn, activates the gp130/JAK signaling pathway. Blockade of this pathway inhibits cell-cycle progression and cell growth and represses tumorigenesis.

\section{Results}

The presence of activated STAT3 correlated positively with adenocarcinomas harboring mutant EGFR ( $\triangle E G F R$ and L858R). Immunohistochemical analysis of tissue microarrays (TMAs) of primary lung adenocarcinomas (92 tumor specimens) showed that 3.3\% contained high $(+3), 17.4 \%$ contained moderate $(+2)$, and $29.3 \%$ contained low $(+1)$ levels of nuclear pSTAT3, while $50.0 \%$ had no $(0)$ PSTAT3 (Figure 1 ). Given the clear distinction between a staining score of 0 and +1 , a score of $+1,+2$, or +3 was regarded as positive, and a score of 0 was regarded as negative. A similar distribution and grading system for pSTAT3 levels has been described by others (14-16). As can be seen in Table 1, with the use of the Fisher exact test and Mann-Whitney $U$ test for statistical analyses, there was a strong correlation between the presence of mutant EGFRs and pSTAT3 $(P=0.002)$. Other positive correlations existed between pSTAT3 and (a) pEGFR, pAKT, and pERK1/2 (pMAPK); (b) decreased expression of caspase-3 protein; (c) non-solid tumor; (d) small tumor size $(\leq 3 \mathrm{~cm})$; and (e) thyroid transcription factor 1 (TTF1) expression. A positive correlation between pSTAT3 levels and tumor size, pEGFR, decreased apoptotic bodies, and well-differentiated tumors has been described previously $(14,15)$. PSTAT3 positivity on immunohistochemical analysis did not correlate with Ki67 protein expression; amplification of the EGFR gene (chromogen in situ hybridization [CISH]); or patient age, sex, cigarette smoking pack year history, and survival (data not shown).

JAK inbibition, but not EGFR inhibition, blocks STAT3 phosphorylation, induces a $G_{2} / M$ cell cycle arrest, and reduces anchorage-independent growth and tumorigenesis in human lung adenocarcinoma-derived cell lines harboring mutant constitutively activated forms of EGFR. To begin exploring the mechanisms responsible for the elevated levels of PSTAT3

\section{Table 1}

Mutant EGFR expression correlates with tyrosinephosphorylated STAT

\begin{tabular}{|c|c|c|c|}
\hline \multirow[t]{2}{*}{ Category } & \multicolumn{2}{|c|}{ pSTAT3 staining } & \multirow[t]{2}{*}{$P$} \\
\hline & 0 & $+1,+2$, or +3 & \\
\hline Mutant EGFR & 2 & 14 & $0.002^{\mathrm{A}}$ \\
\hline WT EGFR & 44 & 32 & \\
\hline pEGFR+ & 2 & 9 & $0.050^{\mathrm{A}}$ \\
\hline pEGFR- & 44 & 37 & \\
\hline EGFR $\mathrm{CISH}^{+}$ & 7 & 12 & $0.300^{A}$ \\
\hline EGFR $\mathrm{CISH}^{-}$ & 39 & 34 & \\
\hline pAKT nuc ${ }^{+}$ & 5 & 26 & $0.000^{A}$ \\
\hline pAKT nuc- & 41 & 20 & \\
\hline pERK1/2 & 46 & 46 & $0.007^{\mathrm{B}}$ \\
\hline Caspase-3 & 46 & 46 & $0.005^{\mathrm{B}}$ \\
\hline TTF1+ & 32 & 41 & $0.038^{A}$ \\
\hline TTF1- & 14 & 5 & \\
\hline Solid tumors & 17 & 6 & $0.015^{\mathrm{A}}$ \\
\hline Other tumors & 29 & 40 & \\
\hline Tumor size $\leq 3 \mathrm{~cm}$ & 11 & 26 & $0.003^{\mathrm{A}}$ \\
\hline Tumor size $>3 \mathrm{~cm}$ & 35 & 20 & \\
\hline
\end{tabular}

A total of 92 tumor specimens were analyzed. $\mathrm{CISH}$, chromogen in situ hybridization; nuc, nuclear staining. ${ }^{A}$ Analyzed with Fisher exact test. BAnalyzed with Mann-Whitney $U$ test. 
A

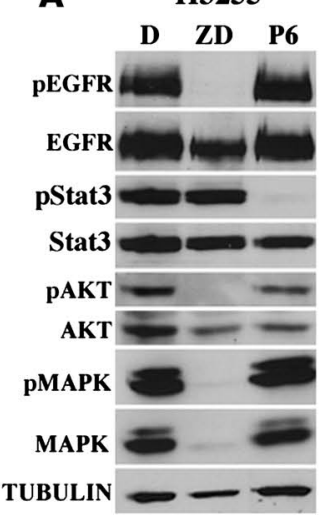

H1975

D ZD P6

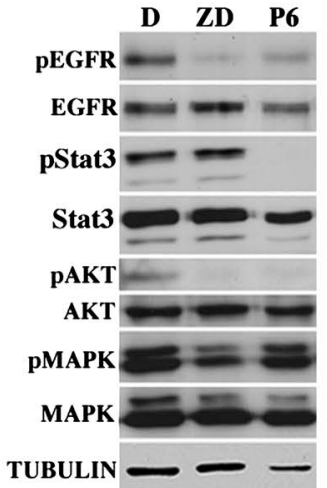

B

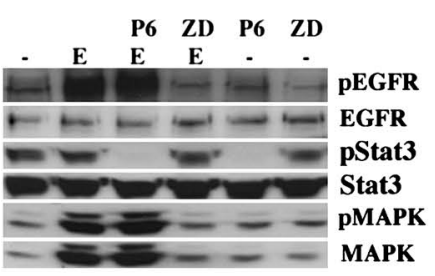

H1650

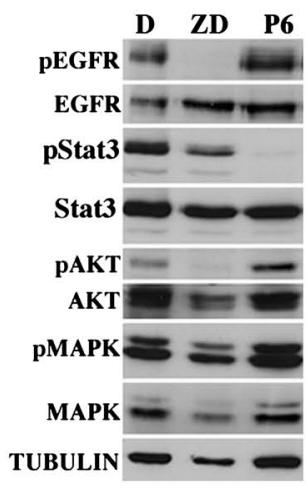

H460
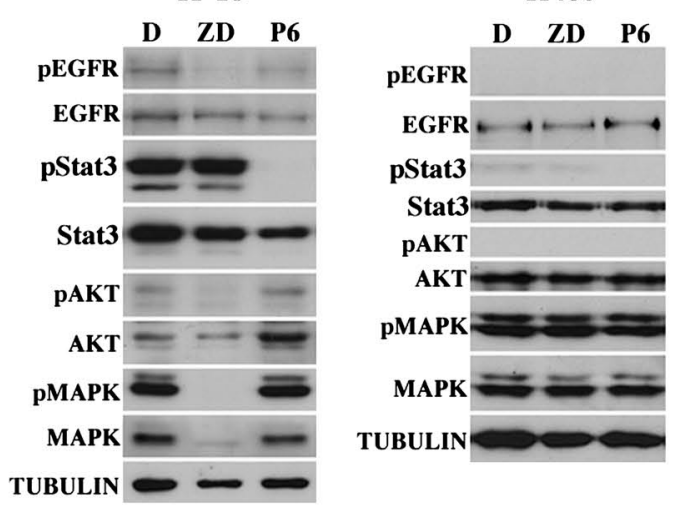

$$
\text { D ZD P6 BMS }
$$

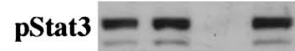

Stat3 - - -
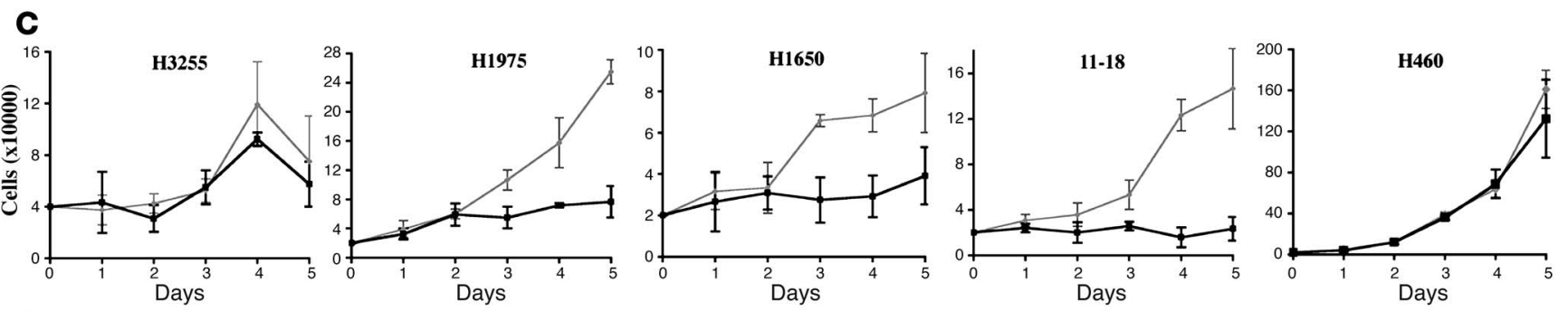

D
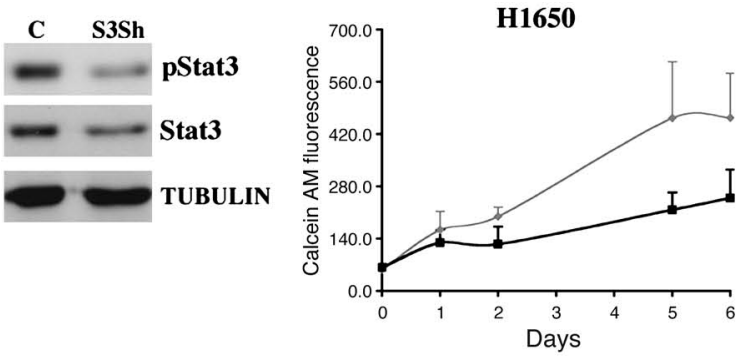

Figure 2

JAK inhibition, but not EGFR inhibition, blocks STAT3 phosphorylation and induces growth arrest in human lung adenocarcinoma-derived cell lines harboring mutant EGFR. (A) Extracts from H3255, H1975, H1650, 11-18, and H460 cell lines, treated with DMSO (D), ZD (5 $\mu$ M), P6 $(1 \mu \mathrm{M})$, or BMS $(5 \mu \mathrm{M})$ for 16 hours, were analyzed for phospho- and total EGFR, STAT3, AKT, and MAPK as well as $\alpha$-tubulin as a loading control by Western blot analysis. (B) Extracts from 11-18 cells, treated with DMSO, P6 (1 $\mu \mathrm{M})$, or ZD (5 $\mu \mathrm{M})$ for 30 minutes and stimulated with EGF (E) $(100 \mathrm{ng} / \mathrm{ml})$ for 10 minutes, were analyzed for pEGFR, EGFR, pSTAT3, STAT3, pMAPK, and MAPK. (C) Cell proliferation of H3255, H1975, $\mathrm{H} 1650,11-18$, and $\mathrm{H} 460$ cells treated with P6 was determined over a 5-day period. Light gray line, DMSO treatment; black line, P6 treatment. (D) Extracts obtained from scrambled control (C) and STAT3 shRNA-expressing (S3Sh-expressing) H1650 cells were analyzed for pSTAT3, STAT3, and $\alpha$-tubulin (left). Proliferation of the H1650 control (light gray line) and H1650 S3Sh (black line) was evaluated by calcein AM.

in mutant EGFR-expressing lung adenocarcinomas, we obtained lung adenocarcinoma-derived cell lines harboring mutant forms of EGFR, which are known to contain constitutively activated STAT3 $(9,14,28)$. As can be seen in Figure 2, the 11-18 (L858R) (29), H1650 (exon 19 deletion, $\Delta 746-750)$, H1975 (L858R), and H3255 (L858R, 11-fold amplification) cell lines all expressed high levels of typrosine-phosphorylated STAT3 (pSTAT3) by Western blot analysis. The exception was noted for control H460 (wild-type EGFR) cells (Figure 2A). The mechanism of STAT3 activation in these cell lines remains unclear, as inhibition of src and JAK2 activity did not affect pSTAT3 levels and inhibition of EGFR activity with the use of ZD reduced pSTAT3 levels in only a few of the cell lines $(14,28)$. We reexamined the role of these kinases in mediating STAT3 phosphorylation in the 4 cell lines described above. We recently described a novel pan-JAK inhibitor (P6) as being more specific and sensitive than AG490 in the treatment of myeloma cells (30). P6 markedly abrogated pSTAT3, while neither EGFR tyrosine kinase inhibition (ZD) nor src inhibition (dasatinib) had 
A $11-18$
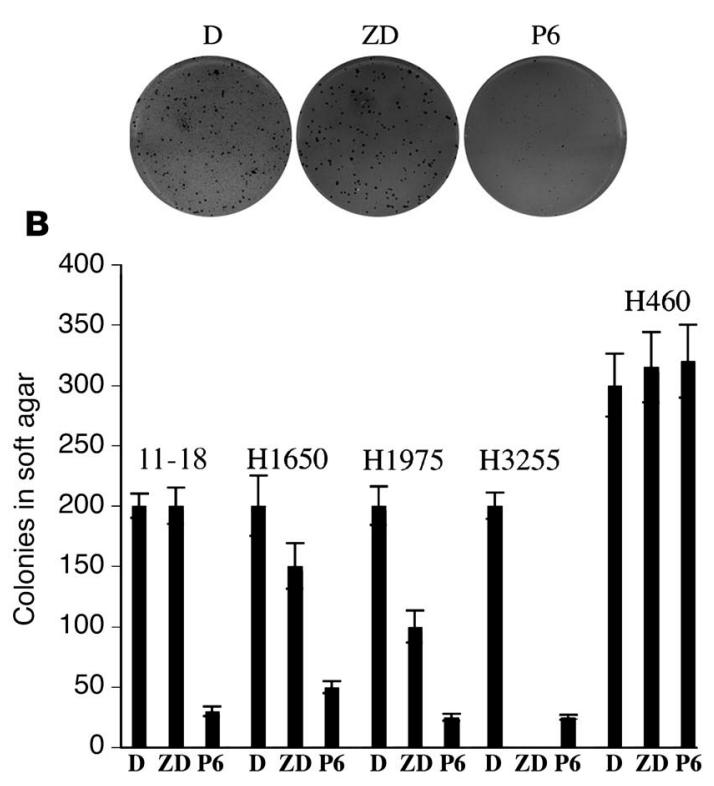

c

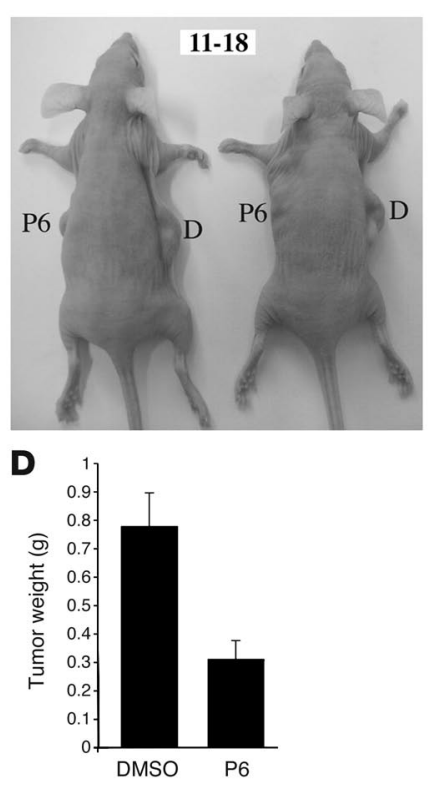

\section{Figure 3}

P6 inhibits tumorigenesis of human lung adenocarcinoma cell lines in vitro and in vivo. (A) 11-18, H1650, H1975, H3255, and H460 cells $\left(5 \times 10^{3} /\right.$ well $)$ were plated in soft agar in the presence of DMSO, ZD, or P6. Colony numbers were counted after 14 days. A representative colony growth of 11-18 is shown with the indicated treatment. (B) Soft agar colony numbers of treated 11-18, H1650, H1975, H3255, and $\mathrm{H} 460$ cell lines are shown (mean $\pm \mathrm{SD}$ ). (C) 11-18 cells were treated with DMSO or P6 for 16 hours and injected into the flanks of nude mice. Size and weight of tumors were determined after 14 days (mean \pm SD). Examples of 2 animals with representative injections are shown. (D) Weight of the tumors from DMSOor P6-treated cells are shown (mean $\pm \mathrm{SD}$ ). any effect on pSTAT3 levels (Figure 2A and data not shown). P6 had a minimal effect on pEGFR levels, and it did not affect any of the 4 important tyrosine phosphorylation sites (i.e., tyrosine 845, 992, 1045, and 1068) on EGFR (Figure 2A and data not shown). P6 had no effect on pMAPK levels in these cell lines, while an effect on pAKT was observed for the H1975 cell line (whose relative pAKT levels are quite low) (Figure 2A). Addition of EGF to the 11-18 and H3225 cells increased pEGFR and pMAPK levels, but it had no effect on pSTAT3 levels (Figure 2B and data not shown). P6 had no effect on pEGFR levels but completely abrogated pSTAT3 levels, while ZD blocked pEGFR but did not affect pSTAT3 (Figure $2 \mathrm{~B}$ and data not shown). These data suggest that the EGF/EGFR pathway is independent of the JAK/STAT3 pathway.

STAT3 is known to support cellular transformation and sustain cell survival in a number of cancer-derived cell lines, including lung cancer $(13,14,28)$. We subsequently determined whether P6induced PSTAT3 inhibition had any effect on cancer cell growth. P6 treatment dramatically repressed cell proliferation of PSTAT3 $^{+}$ 11-18, H1650, and H1975 cells, but not of pSTAT3- H460 cells (Figure 2C). P6 had no effect on cell growth of H3255 cells. FACS analyses of P6-treated 11-18, H1650, and H1975 cells showed a cell-cycle arrest at the $\mathrm{G}_{2} / \mathrm{M}$ phase, while cleaved poly(ADP ribose) polymerase (PARP) levels and TUNEL assays failed to detect any apoptosis in these samples (data not shown). We hypothesize that

\section{Figure 4}

The IL-6/gp130/JAK pathway mediates STAT3 phosphorylation in lung adenocarcinoma-derived cell lines. (A) Extracts isolated from $\mathrm{H} 3255$, 11-18, and $\mathrm{H} 1650$ cell lines treated with control mouse IgG, gp130blocking mAb (B-R3), or IL-6-blocking mAb ( $\alpha$ IL-6) after a medium change were analyzed by Western blotting for pSTAT3, STAT3, and $\alpha$-tubulin as a loading control. (B) CM collected from 11-18 cells was added to MCF-10A cells in the presence of control mouse IgG, B-R3, $\alpha$ IL-6, IL-6R ( $\alpha$ IL-6R), OSM ( $\alpha$ OSM), and LIF ( $\alpha$ LIF) blocking antibodies. Extracts isolated from these treated cells were analyzed by Western blot analysis for PSTAT3 and STAT3. the growth-inhibitory effects from P6 are principally due to blockade of STAT3 activity. We therefore introduced a STAT3 short hairpin RNA (shRNA) construct into the H1650 cells. This led to a partial decrease in STAT3 levels, which was associated with a $50 \%$ reduction in the proliferation of these cells in comparison with findings for scrambled control-infected cells (Figure 2D).

We next tested the effect of P6 on tumorigenesis of these cell lines. Anchorage-independent soft agar assays were performed, and P6 strongly repressed colony formation of all pSTAT3-harboring cell lines 11-18, H1650, H1975, and H3255 but not of non-pSTAT3-harboring H460 cells (Figure 3, A and B). The EGFR TKI ZD inhibited colony growth of $\mathrm{H} 3255$ cells, and partial inhibition was observed in H1975 cells (Figure 3B). To further assess the antitumorigenic effect of P6, 11-18 and H1650 cells were treated with P6 in vitro for 16 hours and subsequently injected subcutaneously into the flanks of nude mice. After 14 days, the P6-treated cells had grown more slowly compared with the control (DMSO-treated) cells, leading to tumors that were smaller in size and mass (Figure 3, C and D).

A

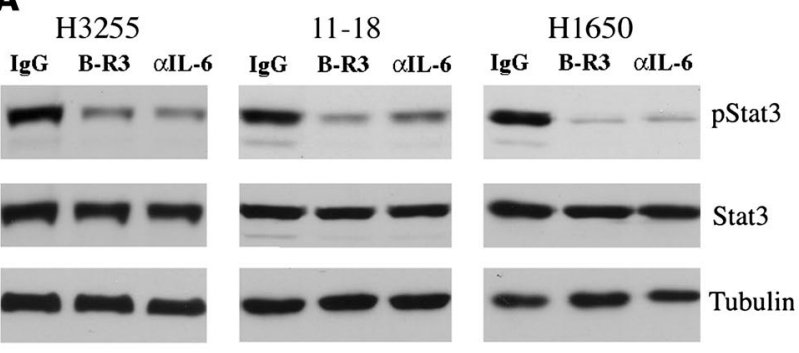

B IgG $\quad$ B-R3 $\alpha$ IL-6 $\alpha$ IL-6R $\alpha$ OSM $\alpha$ LIF

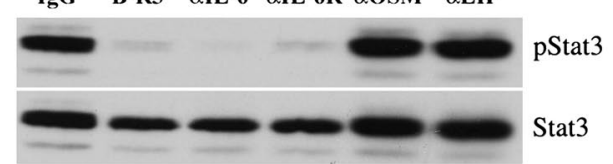


A
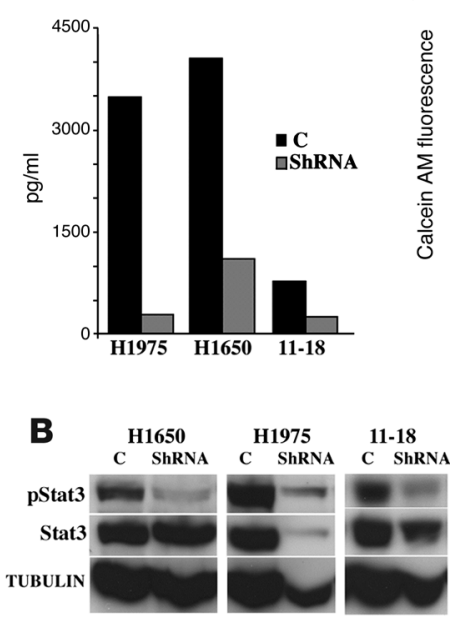

C

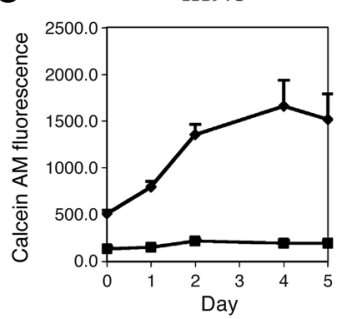

H1650

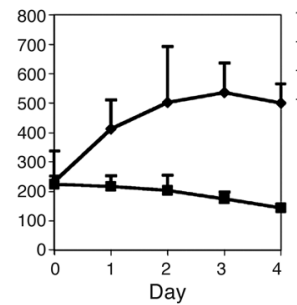

11-18

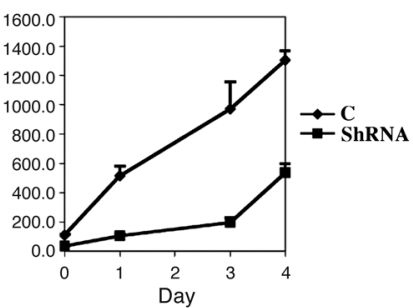

D
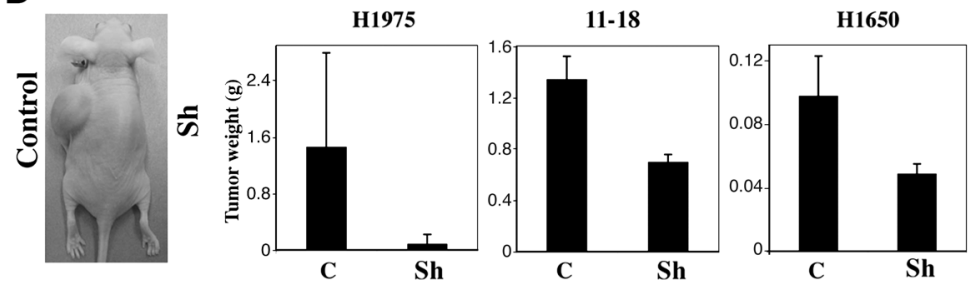

\section{Figure 5}

Blockade of IL-6 signaling with IL-6 shRNA inhibits growth of cell lines. (A) IL-6 shRNA lentivirus (ShRNA) and control lentivirus (C) were introduced into $\mathrm{H} 1975, \mathrm{H} 1650$, and 11-18 cell lines. After 72 hours of selection with puromycin, levels of IL-6 were determined by ELISA of CM. (B) Extracts isolated from the above-described cell lines were analyzed by Western blotting for pSTAT3, STAT3, and $\alpha$-tubulin as a loading control. (C) A total of 2,000 cells/cm² were seeded, and proliferation was determined daily with the use of calcein AM. (D) H1975, 11-18, and H1650 cells, expressing either control or IL-6 shRNA (Sh), were injected into the flanks of nude mice. The tumor weight was determined after 21 days (mean \pm SD) (right). An example of an animal injected with H1975 cells infected with control or IL-6 shRNA is shown (left).

The IL-6/gp130/JAK pathway is responsible for STAT3 phosphorylation in lung adenocarcinoma-derived cell lines. The gp130/JAK pathway signaling has been shown to mediate STAT3 phosphorylation in many cancer-derived cell lines $(22,31-33)$. The IL-6 family cytokines, including IL-6, OSM, and LIF, share the same gp130signaling receptor, leading to activation of JAK1, JAK2, and Tyk2 and subsequent phosphorylation of STAT3 $(21,23)$. To determine whether the IL-6/gp130 signaling pathway was involved in STAT3 activation, we treated the lung cancer-derived cell lines with either a functional blocking antibody to the gp130 receptor or an IL-6sequestering antibody. Treatment with anti-gp130 antibody (B-R3) or anti-IL-6 antibody inhibited PSTAT3 in 11-18, H1650, and $\mathrm{H} 3255$ cells (Figure 4A). To determine whether paracrine production of IL- 6 was responsible for activating the gp130 receptor in these cell lines, conditioned medium (CM) was isolated from 11-18 cells and applied to MCF-10A cells (which do not express pSTAT3) in the absence or presence of various blocking antibodies against gp130, IL-6, IL-6-specific receptor IL-6R, and other gp130 pathway cytokines, OSM and LIF. 11-18 CM induced high levels of PSTAT3, which was inhibited by gp130, IL-6 ligand, or IL-6 receptor antibodies, but not in control IgG-, anti-OSM-, or anti-LIF antibody-treated MCF-10A cells (Figure 4B). We examined mRNA levels for the IL-6 family of cytokines, including $I L-6, O S M, L I F, I L-11$, and CNTF, and only IL-6 mRNA was detected in 11-18, H3255, H1650, and H1975 cell lines (data not shown). IL-6-secreted protein levels were determined by ELISA in the CM collected from near-confluent cell cultures: 11-18, 1,800 pg/ml; H3255, 2,900 pg/ml; H1650, 7,700 $\mathrm{pg} / \mathrm{ml}$; and H1975, 7,800 pg/ml. These levels are high in comparison with the levels of normal cells or other cancer-derived cell lines, for which no more than 10-60 pg/ml are seen (M. Berishaj and J.F. Bromberg, unpublished observations). These findings demonstrate that IL- 6 is being secreted from these cell lines, leading to activation of the gp130/JAK/STAT3 signaling pathway.
Decreased IL-6 blocks PSTAT3 and growth in vitro and in vivo of lung cancer-derived cell lines. To determine the consequences of IL- 6 blockade on growth, we introduced an IL- 6 shRNA construct into the lung cancer-derived cell lines as the blocking antibodies led only to a transient inhibition of IL- 6 signaling. IL-6 shRNAexpressing cell lines produced substantially less IL-6 compared with shRNA control-expressing cells as measured by ELISA of CM (Figure 5A). The consequences of decreased IL-6 production included inhibition of PSTAT3, as well as inhibition of growth in vitro and in vivo (Figure 5, B-D).

$\triangle E G F R$ mediates IL-6 production, STAT3 activation, and cellulartransformation of MCF-10A cells. We wanted to determine whether the expression and the activity of mutant EGFR could regulate IL-6 production. To address this question, we stably introduced and expressed $\triangle E G F R$ in immortalized, but not transformed, breast epithelial MCF-10A cells (Figure 6). The $\triangle$ EGFR-containing cells expressed high levels of $\mathrm{pEGFR}$ and its downstream targets, including PSTAT3 and MAPK, compared with vector control-containing cells (Figure $6 \mathrm{~A}$ ). The $\triangle \mathrm{EGFR}$-expressing cells appeared larger, more irregular, and mesenchymal in nature compared with the "cobblestoned"-appearing control cells (data not shown). $\triangle E G F R$ expressing MCF-10A cells grew in an anchorage-independent manner as well as in the flanks of immunocompromised mice, in contrast to control cells, demonstrating that the sufficiency of $\triangle \mathrm{EGFR}$ in mediating tumorigenesis (Figure 6, B and C). We next determined whether the $\triangle$ EGFR-expressing MCF-10A cells produced high levels of IL-6, leading to activation of STAT3, as was observed in lung adenocarcinoma-derived cell lines harboring mutant EGFR. The $\triangle$ EGFR-MCF-10A cells were treated with P6, gp130, and IL- 6 blocking antibodies, which inhibited STAT3 activation. In contrast, EGFR inhibition (ZD) had no effect on PSTAT3 levels (Figure 7A). RT-PCR analysis revealed high $I L-6$ mRNA expression in $\triangle \mathrm{EGFR}-\mathrm{MCF}-10 \mathrm{~A}$ cells relative to expression in control MCF- 
A

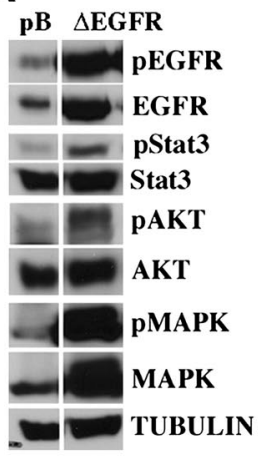

B

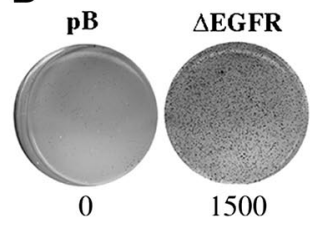

C

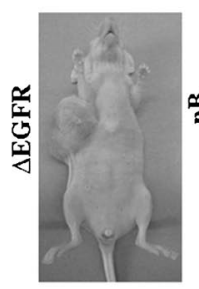

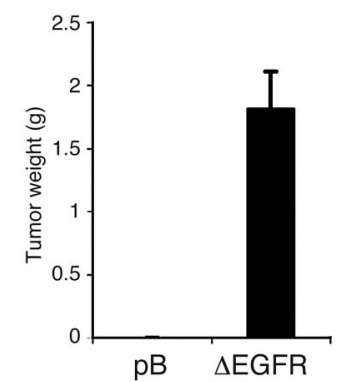

10A cells (Figure 7B). The CM collected from these cells stimulated STAT3 phosphorylation when applied to control MCF-10A cells, while the CM collected from vector control MCF-10A cells did not mediate STAT3 phosphorylation (Figure 7C). As determined by ELISA, $\triangle$ EGFR-MCF-10A cells produced high levels of IL-6 (900 $\mathrm{pg} / \mathrm{ml})$. Levels of OSM, LIF, and gp130 mRNA were also determined and were not elevated (data not shown). These findings suggest that $\triangle$ EGFR expression can upregulate IL- 6 mRNA and protein expression, leading to STAT3 phosphorylation.

EGFR tyrosine kinase inbibition reduces de novo production of IL-6. We demonstrated that transient inhibition of EGFR kinase activity in either cancer-derived cell lines or $\triangle E G F R-M C F-10 A$ cells does not affect pSTAT3 levels (Figure 2A and Figure 7A). However, expression of $\triangle E G F R$ led to increased IL- 6 production, resulting in phosphorylation of STAT3. How do we resolve these 2 observations? The IL-6 protein is stable (34), and we have demonstrated that IL-6 levels were between 900 and $8,000 \mathrm{pg} / \mathrm{ml}$ in CM derived from cell lines expressing mutant EGFR. As little as $50-100 \mathrm{pg} / \mathrm{ml}$ can mediate STAT3 phosphorylation in MCF-10A cells (data not shown). We hypothesized that transient inhibition of IL-6 production through EGFR kinase blockade would have no effect on pSTAT3 levels in cells bathed in high levels of IL-6-containing media. To examine the contribution of EGFR activity on IL- 6 production, the existing IL-6 had to be removed. To test our hypothesis, we replaced the CM with fresh medium, washing away the existing IL-6 and

\section{Figure 7}

Overexpression of $\triangle E$ EFFR mediates IL- 6 production of MCF-10A cells. (A) Extracts isolated from $\triangle$ EGFR MCF-10A cells treated with DMSO, ZD, P6, B-R3, or $\alpha$ lL- 6 for 16 hours were analyzed by Western blotting for pEGFR, EGFR, pSTAT3, STAT3, pAKT, pMAPK, and MAPK. The last lane was run on the same gel but was noncontiguous. (B) Human IL-6 mRNA levels from MCF-10A control (pB) and $\triangle$ EGFR MCF-10A cells were determined by RT-PCR and normalized to $\beta$-actin. (C) Extracts were isolated from MCF-10A cells treated with CM from control cells $(\mathrm{pB})$ and $\triangle$ EGFR MCF-10A cells and analyzed by Western blotting for PSTAT3 and STAT3.

\section{Figure 6}

Overexpression of $\triangle E G F R$ protein in MCF-10A cells induces persistent phosphorylation of STAT3, AKT, and MAPK as well as tumorigenesis. (A) Extracts isolated from control pBabe (pB) and $\triangle E G F R$-expressing MCF-10A cells were analyzed by Western blot for phospho- and total EGFR, STAT3, AKT, and MAPK as well as $\alpha$-tubulin as a loading control. The lanes were run on the same gel but were noncontiguous. (B) Soft agar colony formation assays for MCF-10A control pB and $\triangle$ EGFR-expressing cells are shown. Colony numbers are shown below. (C) MCF-10A control pB and $\triangle$ EGFR-expressing cells were injected into the flanks of nude mice. No tumor was detected with $\mathrm{pB}$, while $\triangle$ EGFR-expressing MCF-10A cells formed tumors. Tumor weight was determined after 21 days (mean $\pm S D$ ) (right). An example of an animal injected with MCF-10A- $\Delta$ EGFR-expressing cells is shown (left).

treating cells with ZD, the EGFR TKI. De novo IL-6 production was inhibited in 11-18 and H1650 cells treated with ZD compared with DMSO control. In contrast, IL- 6 levels from cells that did not undergo a medium change were unaffected by ZD treatment (Figure 8A). Of importance, pSTAT3 levels were inhibited only in cells that had undergone a medium change with $\mathrm{ZD}$, in contrast with no medium change and ZD (Figure 8B). IL-6 production is regulated at multiple levels, including transcription, mRNA stability, posttranslational modification, and secretion. We examined the possible contribution of EGFR activity to the transcriptional control of the $I L-6$ gene. We first determined that treatment of 11-18 cells for 16 hours with ZD, preceded by a medium change, resulted in a decrease in IL- 6 mRNA levels, as determined by semiquantitative RT-PCR and real-time PCR (Figure 8C). In contrast, $I L-6$ mRNA levels were unchanged in cells that had not undergone a medium change before the addition of $\mathrm{ZD}$ (data not shown). Finally, we examined the effect of $\triangle E G F R$ expression and activity on luciferase activity in an $I L-6$ promoter luciferase reporter assay. pGL3-IL-6-Luc contains 2,120 bp of promoter sequence with known binding sites for transcriptional factors such as $\mathrm{C} / \mathrm{EBP} \beta, \mathrm{CREB}, \mathrm{AP} 1$, and NF- $\kappa \mathrm{B}(35)$. Cotransfection of $\triangle \mathrm{EGFR}$ and this IL- 6 promoter luciferase construct into NIH3T3 cells revealed an increase in luciferase activity, which was repressed by $50 \%$ with $\mathrm{ZD}$ (Figure 8D). These results support the suggestion that $\triangle$ EGFR activity can, in part, mediate transcriptional activation of
A

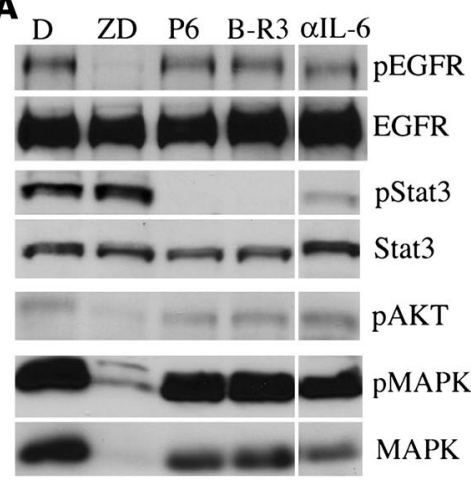

B

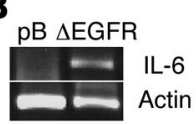

C

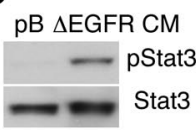



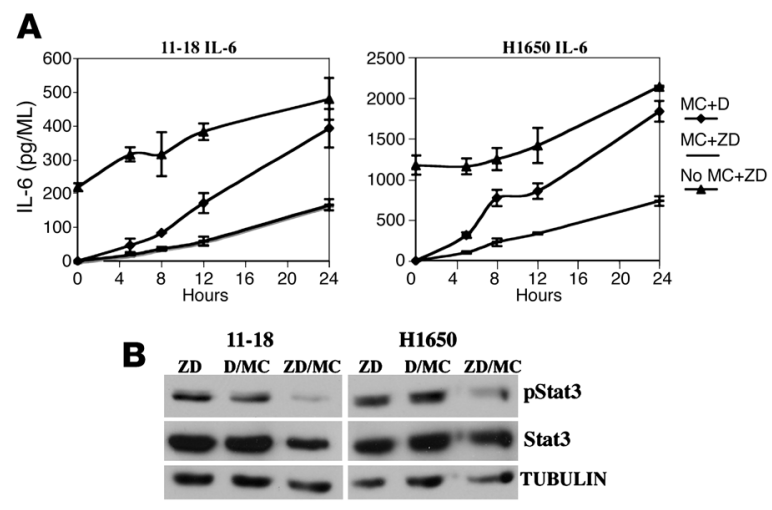

H1650
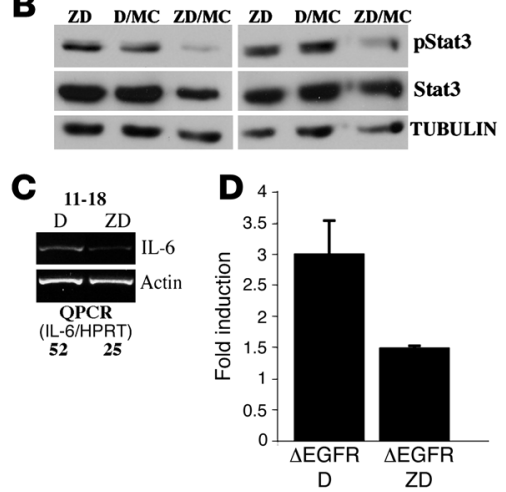

the IL- 6 gene and the subsequent production of IL- 6 protein. The transcriptional regulation of the $I L-6$ gene is complex, involving multiple transcription factors, and our data suggest that other factors (possibly present in CM) can mediate its regulation. Nevertheless, our data suggest that overexpression of mutant EGFR protein may be the initiating step in the production of IL-6.

pSTAT3 levels correlate positively with IL-6 expression in primary lung adenocarcinomas. Given that the apparent mechanism of STAT3 phosphorylation is through the IL-6/gp130/JAK pathway in lung cancer-derived cell lines harboring EGFR mutations, we sought to determine whether IL-6 levels would correlate with pSTAT3 levels in primary lung cancer specimens. Immunohistochemical analysis of the previously described TMAs of primary lung adenocarcinomas revealed that $7.6 \%$ contained high $(+3), 58.7 \%$ contained moderate $(+2)$, and $28.3 \%$ contained low $(+1)$ levels of IL- 6 , while

\section{Figure 8}

EGFR tyrosine kinase inhibition reduces de novo production of IL-6. (A) 11-18 and $\mathrm{H} 1650$ cells were treated with a medium change (MC) with ZD $(M C+Z D)$, no MC with ZD (No MC+ZD), or MC with DMSO $(M C+D)$. Levels of IL- 6 were measured by ELISA at the indicated times after the addition of ZD (mean $\pm S D$ ). (B) Shown are extracts isolated from $11-18$ and $\mathrm{H} 1650$ cells treated as described above after 16 hours and analyzed for pSTAT3, STAT3, and $\alpha$-tubulin. (C) Human IL-6 mRNA levels from 11-18 cells treated with DMSO or ZD for 16 hours after a medium change were determined by RT-PCR and normalized to $\beta$-actin. The same mRNA samples were analyzed by quantitative real-time PCR (QPCR), and the IL-6 mRNA levels (normalized to hypoxanthine-guanine phosphoribosyltransferase [HPRT]) are shown below. (D) NIH3T3 cells were cotransfected with an IL-6 reporter construct, a TK-Renilla construct (for transfection/loading control), and either a $\mathrm{pB}$ vector (baseline activity) or a $\mathrm{pB}-\Delta \mathrm{EGFR}$ expression construct. Twenty-four hours after transfection, DMSO or ZD was added, and an additional 24 hours later, cells were lysed and subjected to firefly and Renilla luciferase activity measurements. The bars show fold induction over the baseline activity (mean $\pm \mathrm{SD}$ ).

\section{Figure 9}

pSTAT3 levels correlate positively with IL-6 expression in primary lung adenocarcinomas. Immunohistochemical analysis of TMAs of primary lung adenocarcinomas (92 tumor specimens) for IL-6 were scored as $0(5 / 92),+1$ $(26 / 92),+2(54 / 92)$, or +3 (7/92). Examples of selected tumor specimens from sequential sections of the same TMAs stained for IL-6 (top, left to right, $0,+1,+3$, and +3 ) and pSTAT3 (bottom, left to right, 0 , $0,+3$, and +3 ) are shown. A positive correlation was observed in specimens that were PSTAT3 positive $(+1,+2$, or +3$)$ and expressing moderate-to-high $(+2$ to +3$)$ levels of IL- 6 as determined by analysis with the Fisher exact test $(P<0.001)$.
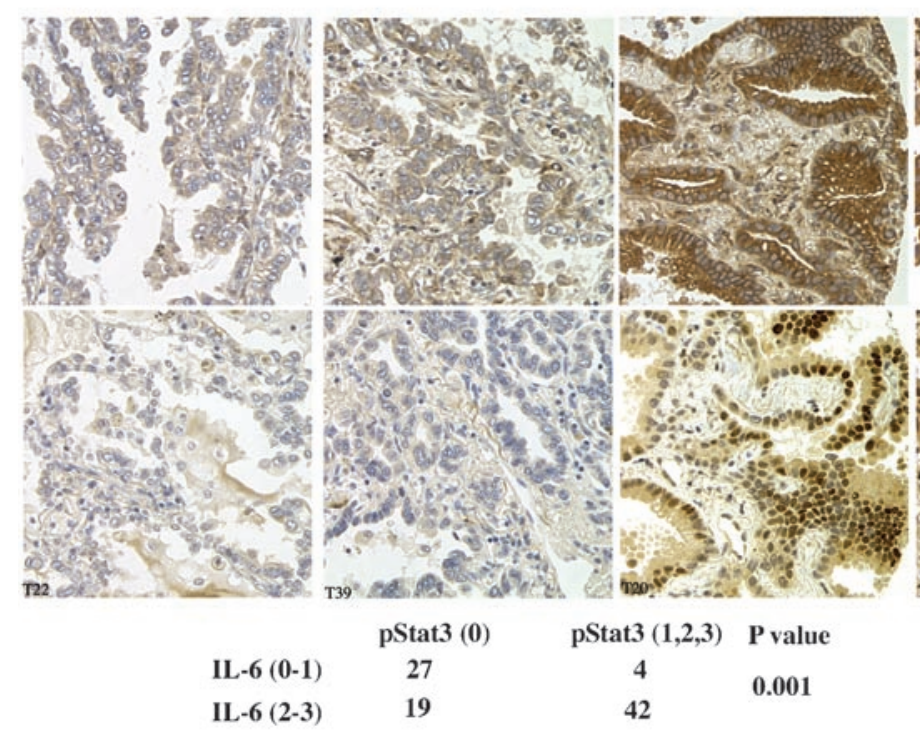

$5.4 \%$ had no (0) IL-6. A previous analysis of 10 primary lung tissue samples revealed that $90 \%$ expressed high levels of IL-6, which is consistent with our observations (36). In the current work, a strong positive correlation was observed in lung specimens that were PSTAT3 positive (staining score of $+1,+2$, or +3 ) and expressing moderate-to-high levels (staining score of +2 to +3 ) of IL- 6 $(P<0.001)$ (Figure 9). In Figure 9, examples of selected tumor specimens from the same TMAs stained for IL-6 (top) and pSTAT3 (bottom) are shown. These findings strongly underscore the contribution of IL- 6 in persistent activation of STAT 3 in both primary lung adenocarcinomas and cancer-derived cell lines.

\section{Discussion}

Identification of potential factors and regulatory pathways mediating STAT3 activation has important implications for the develop- 
ment and selection of molecularly targeted therapy in lung cancer. Our current findings demonstrate that the presence of PSTAT3 in lung adenocarcinoma tumor samples by immunohistochemical analysis correlates positively with expression of EGFR tyrosine kinase domain mutations. Owing to insufficient numbers of tumor specimens expressing somatic-activating mutations within the kinase domain, we were unable to determine whether PSTAT3 positivity correlated differentially with either $\triangle E G F R$ or L858R. Much of our data confirms prior observations regarding PSTAT3 and lung adenocarcinomas. Specifically, 50\% of samples express variable levels of pSTAT3 by immunohistochemical analysis; a positive association exists between pSTAT3 and small tumor size, TTF1, pAKT, and pEGFR; and an inverse correlation exists between pSTAT3 and caspase-3 (14-16). No association between pSTAT3 and survival was observed. A recent description of a murine model revealed inducible expression of EGFR kinase domain-activating mutations targeted to the lung epithelium gives rise to adenocarcinomas containing PSTAT3 and PAKT, demonstrating an association between this oncogene and activated STAT3 (26). Of interest, expression of constitutively activated STAT3 targeted to the lung epithelium gives rise to de novo adenocarcinoma of the lung (C. Yan, unpublished observations).

The evidence demonstrating a direct association between EGFR and STAT3 is based primarily on work done in cell lines expressing high levels of EGFR, such as A431 and head and neck squamous cell carcinoma (HNSCC) cells $(37,38)$. The biochemical differences between cell lines expressing kinase domain-activating mutations within the EGFR versus wild-type EGFR include enhanced differential phosphorylation of specific tyrosine residues on the EGFR and alterations in the levels of pMAPK, pAKT, p-src, and PSTAT3 $(8,9,28,39,40)$. However, the mechanism of STAT3 phosphorylation in either mutant EGFR-transfected cell lines or cancerderived cell lines is unclear, as inhibition of EGFR (with ZD), src (with dasatinib), or JAK2 (with AG490) activity does not lead to inhibition of STAT3 phosphorylation in cancer-derived cell lines $(8,14,28)$. Our current findings demonstrate that inhibition of JAKs with the use of a pan-JAK inhibitor (P6) completely abrogated STAT3 phosphorylation in all cancer-derived cell lines expressing mutant forms of EGFR (Figure 2). Of importance, inhibition of neither EGFR activity (with ZD) nor src activity (with dasatinib-BMS) resulted in a change in pSTAT3 levels (Figure 2A and data not shown). The use of a pan-JAK inhibitor, rather than an inhibitor specific for only a single JAK, is likely critical, as multiple JAKs could be implicated in mediating STAT3 phosphorylation in an EGFR-dependent manner. The JAKs have been described as being associated with EGFR, but they are not required to mediate STAT phosphorylation in response to EGF (41). P6 treatment of mutant EGFR cancer-derived cell lines did not markedly affect EGFR phosphorylation or PMAPK, which is a target of the EGFR (Figure 2A). Furthermore, the addition of EGF to these cell lines increases pEGFR, which was unaffected by P6 treatment (Figure $2 \mathrm{~B}$ and data not shown). These data suggest an uncoupling between EGFR and JAKs.

Identification of the regulatory pathways leading to STAT3 activation has clear implications for the development of targeted therapy in lung cancer (13). Of importance, we demonstrate that inhibition of JAK kinases with the use of the pan-JAK inhibitor P6 led to decreased proliferation and $\mathrm{a}_{2} / \mathrm{M}$ cell cycle block in 11-18, H1975, and H1650 cell lines (Figure 2C and data not shown). We demonstrate that STAT3 is likely the principal P6 target, as a par- tial knockdown of this protein in the $\mathrm{H} 1650$ cells led to a decrease in their proliferative capacity (Figure 2D). Inhibition of anchorageindependent growth by $\mathrm{P} 6$ was observed in all 4 cell lines, while ZD inhibited growth only in soft agar to comparable levels seen with P6 in H3255 cells (Figure 3, A and B). The H1975 cell line is resistant to the growth-inhibitory actions of $Z \mathrm{D}$ and dasatinib (8). However, our data reveal that $\mathrm{ZD}$, at a relatively higher concentration $(5 \mu \mathrm{M})$, can inhibit the EGFR tyrosine phosphorylation and partially inhibit anchorage-independent growth of H1975 cells (Figure 2A and Figure 3B). This result is consistent with prior observations that $\mathrm{ZD}$, at concentrations ranging from 5 to $10 \mu \mathrm{M}$, inhibits cell growth of H1975 (40). As P6 is insoluble, we could not administer it in vivo. Thus, subsequently, we were only able to show that $\mathrm{P} 6$-treated cells in vitro are less capable of growing in vivo compared with DMSO-treated control cells (Figure 3, C and D). Nevertheless, these findings suggest that JAK inhibition may prove to be effective therapy in the treatment of cancers that depend on mutant EGFR activity, including those that may be less sensitive or resistant to TKIs.

Activation of JAKs and STAT3 has been shown to occur through binding of the IL- 6 family of cytokines to the gp130 receptor in many different cancer-derived cell lines, including myeloma; HNSCC; breast and prostate cancers; cholangiocarcinoma; and NSCLC $(22,24,31-33,42,43)$. Furthermore, high circulating levels of IL- 6 have been found in patients who have metastatic breast and lung cancers $(44,45)$. Our current findings demonstrate that blockade of IL-6/IL-6R/gp130 signaling with the use of both sequestering antibodies and blocking antibodies inhibits STAT3 activation (Figure 4A). Furthermore, all cell lines produce very high levels of IL-6, which was rapidly secreted into the culture medium and could stimulate STAT3 phosphorylation in MCF$10 \mathrm{~A}$ cells (Figure 4B). The degree of PSTAT3 inhibition with the blocking antibodies was not as profound as with P6 treatment. Furthermore, the blockade with both gp130 and IL-6 was only transient, lasting less than 24 hours (data not shown) (33). To examine the consequences of IL- 6 blockade on growth, we introduced an IL- 6 shRNA construct into the lung cancer-derived cell lines. We demonstrated that reduced expression of IL- 6 led to a decrease in PSTAT3, as well as a decrease in the in vitro and in vivo growth of H1975, H1650, and 11-18 cells (Figure 5). Despite reduced production of IL-6, there was no effect on in vitro growth in the H3255 cell line, which is consistent with the lack of growth inhibition by P6 in this cell line (data not shown). Thus, blockade of the IL-6 pathway is sufficient to inhibit the growth of these cancer-derived cell lines and demonstrates the potential therapeutic benefit of IL- 6 inhibition.

Given the previously described association between the EGFR family members and the gp130 receptor, we attempted but were unable to show any physical relation between EGFR and gp130 in H3255 (which expresses the highest levels of EGFR), 11-18, H1975, and $\mathrm{H} 1650$ cell lines (data not shown) $(42,46,47)$. To examine the relation between mutant EGFR and IL-6/gp130 receptor signaling, we introduced $\triangle E G F R$ into MCF-10A cells, and our current findings demonstrate an increased production of IL- 6 mRNA and protein, with subsequent STAT3 activation and cellular transformation (Figure 6). An immortalized breast epithelial cell line was chosen, as introduction of mutant EGFR into immortalized lung epithelial cells has resulted in an increase in pSTAT3 levels and anchorage-independent growth but no evidence of tumorigenesis (48). As observed with the lung cancer-derived cell lines, STAT3 
phosphorylation depends on IL-6/gp130/JAK signaling, but not on EGFR kinase activity in the $\triangle$ EGFR MCF-10A cells (Figure 7). These novel findings suggest that $\triangle \mathrm{EGFR}$ can drive expression of IL-6, which ultimately is responsible for mediating gp130/ JAK/STAT3 signaling.

If mutant EGFR is capable of upregulating IL-6 synthesis, why is there no effect on pSTAT3 levels after inhibition of EGFR activity (Figures 2 and 6)? The IL-6 protein is produced to high levels and is quite stable within the $\mathrm{CM}$ of these cancer-derived cell lines. Thus, decreasing its synthesis in the context of such excess would clearly have little to no consequence for STAT3 activation. To examine the contribution of mutant EGFR activity on IL- 6 production and STAT3 activation, we first had to remove the IL-6-containing CM. After removal of CM, inhibition of the EGFR activity led to a partial decrease in IL- 6 production and PSTAT3. This clearly demonstrates a requirement for mutant EGFR activity, but nonetheless supports the suggestion that other factors are mediating the regulation of IL-6 synthesis (Figure 8, A and B). Some of the described transcriptional mediators of the $I L-6$ gene include NF- $\mathrm{B}, \mathrm{AP}-1, \mathrm{C} / \mathrm{EBP} \beta$, and $\mathrm{CREB}$, whose expressions and activity vary as a function of cell type and ligand stimulation $(35,49-51)$. The EGF has been shown to activate NF- $\mathrm{KB}$ and AP-1 transcription factors in HNSCC-derived cell lines (52). It has been suggested that STAT3, in association with

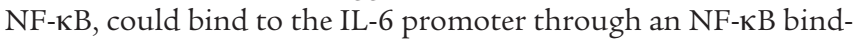
ing site and promote expression of the IL- 6 gene (53). Of interest, IL-6 production has been shown to depend on activated STAT3 in a mouse gastric model system (54). We hypothesize that mutant EGFR leads to NF- $\mathrm{KB}$ activation and that STAT3 can potentiate IL-6 transcription through this critical interaction.

Our cell line data demonstrate a positive relation among mutant EGFR, IL-6, and pSTAT3. We examined lung adenocarcinoma TMAs for IL-6 expression and demonstrate a positive association between pSTAT3-positive samples (i.e., those with a staining score of $+1,+2$, or +3 ) and samples expressing moderate-to-high ( +2 to $+3)$ levels of IL-6. In summary, we demonstrate that the mechanism by which STAT3 is activated in lung adenocarcinomas is through mutant EGFR regulating expression of the IL- 6 cytokine, which, in turn, activates the gp130/JAK pathway. Of importance, JAK kinase and IL- 6 blockade leads to inhibition of growth and tumorigenesis, suggesting that the development of inhibitors of this pathway may be an important therapeutic target for these malignancies.

\section{Methods}

Cell culture, cell growth assays, growth in soft agar, and inhibitors. Human mutant EGFR-expressing NSCLC adenocarcinoma cell lines 11-18, H1650, H1975, and H3255 and control wild-type EGFR cell line H460 were cultured in RPMI-1640 medium with 10\% FBS. The MCF-10A cells (obtained from ATCC) were cultured in $10 \mathrm{~A}$ medium as previously described (55). The NIH3T3 cells were cultured in DMEM containing $10 \%$ FBS. To measure cell growth, $2 \times 10^{4}$ to $4 \times 10^{4}$ cells per well $\left(2 \times 10^{3}\right.$ to $\left.4 \times 10^{3} / \mathrm{cm}^{2}\right)$ were plated into 6-well dishes. Ten hours later, P6 $(1 \mu \mathrm{M})$ or control DMSO was added (day 0 ). Cells were counted at days 1, 2, 3, 4, and 5. Each data point represented the mean value from triplicate wells. In addition, viability assays were performed with the use of calcein AM (acetoxymethylester of calcein; Molecular Probes; Invitrogen) according to the manufacturer's instructions. Cells $\left(2.5 \times 10^{4}\right.$ to $\left.5.0 \times 10^{4}\right)$ were plated in $0.35 \%$ agar in culture medium with DMSO, ZD $(5 \mu \mathrm{M})$, or P6 $(1 \mu \mathrm{M})$. Fresh drug was added to cells every 4 days. Colony number was assessed after 14 days by staining with MTT $(0.5 \mathrm{ml}$ of $5-\mathrm{mg} / \mathrm{ml}$ concentration). P6 (tetracyclic pyridone 2-tert-butyl-9-fluoro-3,6-dihydro-7H-benz[h]-imidaz[4,5-f]isoquinoline-7- one) was synthesized by in our laboratory (56). ZD was a gift from Astra Zeneca, provided by Jackie She of the Anti-Tumor Assessment Core Facility, MSKCC. Dasatinib (BMS-354825) was synthesized in our laboratory.

Retroviral and lentiviral infections and stable cell line establishment. $\mathrm{pBabe-}$ puro and pBabe-EGFR- $\Delta 747-752$ were introduced into MCF-10A cells by retroviral infection and selected with puromycin $(2 \mu \mathrm{g} / \mathrm{ml})$, as previously described (57). IL-6 shRNA lentiviral construct and control lentiviral constructs were purchased from Open Biosystems, and infections into lung cancer-derived cell lines, followed by selection with puromycin $(2 \mu \mathrm{g} / \mathrm{ml})$, were performed according to the manufacturer's instructions. Given that cell growth was inhibited by the IL- 6 shRNA, proliferation assays were conducted and in vivo growth was determined within 72 hours of puromycin selection. STAT3 shRNA and control lentiviral constructs were generated as previously described (58).

DNA constructs. pBabe-EGFR- $\Delta 747-752$ (7), a mutant human EGFR expression construct, was generated by subcloning a wild-type EGFR cDNA into the Xhol site of pBluescript. A PCR-based mutagenic deletion of amino acids 747-752 was subsequently performed using DNA polymerase Pfu and primer sets: $5^{\prime}$-CCCGTCGCTATCAAGGAACCGAAAGCCAACAAGG-3' ' 5'-CCTTGTTGGCTTTCGGTTCCTTGATAGCGACGGG-3'. The mutated EGFR was cut out of the pBluescript with the use of XhoI and ligated to the SalI site of the retroviral construct pBabe-puro (59).

Cell lysate preparation and Western blot analysis. Adherent cells were washed with PBS and lysed in whole-cell lysis buffer $(150 \mathrm{mM} \mathrm{NaCl}, 10 \%$ glycerol, 1\% IGEPAL, $0.5 \%$ sodium deoxycholate, $2 \mathrm{mM}$ EDTA, $1 \mathrm{mM} \mathrm{NaF}, 1 \mathrm{mM}$ $\mathrm{Na}_{3} \mathrm{VO}_{4}, 1 \mathrm{mM} \mathrm{Na} \mathrm{MoO}_{4}, 0.1 \%$ SDS, $20 \mathrm{mM}$ Tris-HCl, pH 8) with proteinase inhibitors. Western blot analysis was performed as previously described (60). Antibodies used for Western blot analysis were as follows: anti-phospho-STAT3 (Tyr705), total STAT3, phospho-MAPK (p44/42, Thr202/ Tyr204), phospho-AKT (Ser473), and total AKT (Cell Signaling); antiMAPK (ERK2), EGFR, and HRP-conjugated anti-rabbit IgG (Santa Cruz Biotechnology Inc.); anti-phospho-tyrosine (4G10) (Upstate Technologies); anti- $\alpha$-tubulin (Sigma-Aldrich); and HRP-conjugated anti-mouse IgG (Jackson ImmunoResearch Laboratories Inc.). For cytokine- and gp130-blocking experiments, cells were treated with blocking antibodies $(5 \mu \mathrm{g} / \mathrm{ml})$ for 16 hours. Human IL-6-blocking antibodies B-E8 and 522 were obtained from Cell Science and R\&D Systems. Human gp130-blocking antibody B-R3 was obtained from Cell Science. IL-6R-, LIF-, and OSMblocking antibodies were obtained from R\&D Systems.

Luciferase reporter assays. pGL3-IL-6-Luc, an IL-6 promoter luciferase reporter construct, was kindly provided by Espen Spangenburg (University of Maryland, College Park, Maryland, USA) (35, 61). The pGL3-IL-6-Luc, a normalization construct TK-Renilla, and either control vector pBabe-puro or pBabe-EGFR- $\Delta 747-752$ were cotransfected into NIH3T3 cells with the use of Lipofectamine 2000 reagent (Invitrogen). The next day, the medium was changed, and DMSO (5 $\mu \mathrm{l} /$ well) or ZD (5 $\mu \mathrm{M})$ was added. Twenty-four hours later, measurements of firefly and Renilla luciferase activities in cell lysates were carried out with the use of the Dual-Luciferase Reporter Assay System (Promega). Data were presented as the ratio of firefly luciferase activity to Renilla luciferase activity in each sample performed in triplicate \pm SD.

ELISA. Cells were grown to approximately $50 \%-85 \%$ confluence, and medium was aspirated. Cells were subsequently washed twice with PBS, and fresh medium was added. At various time points, CM was analyzed for IL-6 with the use of an IL-6 ELISA kit (Cell Science) according to the manufacturer's instructions.

$R T-P C R$. Total cell RNA was extracted from cells by using an RNeasy kit (QIAGEN). Two micrograms of total RNA was used for IL-6 and $\beta$-actin RT-PCR using an iScript RT-PCR kit (Bio-Rad) according to the manufacturer's instructions. Sequences of primers for amplification of the IL-6 gene were as follows: forward primer, 5'-TAGCCGCCCCACACAGACAG- 
3'; reverse primer, 5'-GGCTGGCATTTGTGGTTGGG-3'. The $\beta$-actin primers were as follows: forward primer, 5'-CGTGCGTGACATTAAGGAGA-3'; reverse primer, 5'-TGATCCACATCTGCTGGAAG-3'. The same cDNA samples were also analyzed for real-time quantitative PCR using a Bio-Rad IQ5 Multicolor Real-Time PCR Detection System/SyBR.

Flow cytometry. Cells were resuspended in $0.5 \mathrm{ml}$ of ice-cold 1\% BSA-PBS and fixed with $70 \%$ ethanol. The fixed cells were subsequently washed 3 times with BSA-PBS, resuspended in $1 \mathrm{ml}$ of BSA-PBS containing propidium iodide $(5 \mu \mathrm{g} / \mathrm{ml})$ and $\mathrm{RNase} \mathrm{A}(100 \mu \mathrm{g} / \mathrm{ml})$, and incubated at $37^{\circ} \mathrm{C}$ for 15 minutes. Single-cell suspensions were obtained by filtering cells through a cell strainer for flow cytometry analysis.

Tumorigenesis studies. All animal work was done in accordance with a protocol approved by the MSKCC Institutional Animal Care and Use Committee. Female homozygous athymic nu/nu mice (National Cancer Institute) were used for xenografting studies. After being treated with DMSO or P6 for 16 hours, 11-18 and H1650 cells were harvested. They were subsequently mixed with an equal volume of Matrigel (BD Biosciences) and injected subcutaneously into the flanks of 45-day-old female nude mice. Both tumor size and tumor weight were determined after 14 days. Four animals were used for each experimental group. Control shRNA- and IL-6 shRNA-infected 11-18, H1650, and H1975 cell lines were harvested, and $0.5 \times 10^{6}$ cells were mixed with an equal volume of Matrigel and injected subcutaneously into the flanks of 45-day-old female nude mice. Both tumor size and tumor weight were determined after 21 days. Four animals were used for each experimental group. MCF-10A control pBabe and $\triangle$ EGFR-expressing cells $\left(1 \times 10^{7}\right.$ cells $)$ were mixed with an equal volume of Matrigel and injected subcutaneously into the flanks of 45-day-old female nude mice. Both tumor size and tumor weight were determined after 21 days. Four animals were used for each experimental group.

TMAs and immunohistochemistry. Multitissue blocks of formalin-fixed, paraffin-embedded NSCLC tissue (containing 3 or 4 representative $0.6-\mathrm{mm}$ cores) were prepared using a tissue arrayer, and immunohistochemistry was performed, as described previously (60). Antigen retrieval, with the use of citric acid ( $\mathrm{pH} \mathrm{6.0)}$ at $97^{\circ} \mathrm{C}$ for 30 minutes, was followed by treatment with $3 \%$ hydrogen peroxide. pSTAT3 (Tyr705), pEGFR (pY1068), phospho-AKT, pERK1/2 (pMAPK), and caspase-3 antibodies (Cell Signaling) were used at 1:200, 1:100, 1:50, 1:100, and 1:300 dilutions, respectively. Anti-TTF1 mAb (Dako) was used at a 1:100 dilution. Anti-human IL-6 goat polyclonal anti- body (R\&D Systems) was used at a 1:100 dilution (62). Secondary reagents were from DakoCytomation EnVision+ Dual Link System-HRP (DAB+) kit or the DakoCytomation LSAB+ system-HRP kit. Counterstaining was performed with the use of hematoxylin. Scoring of the TMAs was performed by 2 independent observers, with a high correlation observed between scorers $(P<0.001)$ for both pSTAT3 and IL-6. For a tumor to be considered positive for either PSTAT3 or IL-6, all 4 replicates in the tissue array had to have a similar staining intensity. Otherwise, it was excluded. Statistical analyses were done using StatView (SAS Institute). The correlation between the scores of both scorers and the relation between those of PSTAT3 and IL- 6 were measured by using the $\chi^{2}$ test.

Mutation analysis. DNA samples were extracted from frozen tissue after microdissection. For $E G F R$, restriction fragment alterations were also used to detect the most common point mutation in exons 19 and 21 (63). After screening was completed, the mutated cases were confirmed by sequencing. For samples with only RNA available, RT-PCR was used with the primers listed above, followed by direct sequencing. Di-deoxy sequence analysis of mutational hot spots for exons 18-22 of EGFR was performed with the use of specific primers listed as follows: EGFR-forward, $5^{\prime}$-CCATCGCCACTGGGATG-3'; EGFR-reverse, 5'-TCTTGACATGCTGCGGTG-3'.

\section{Acknowledgments}

This work was supported by NIH grant R01 CA87637, a Patrick M. Byrne grant, the Sussman Family Fund, the Charles and Marjorie Holloway Foundation, the Breast Cancer Alliance, and Lerner and Leigh Awards (to J.F. Bromberg) as well as New York State Department of Health grant C021338 (to S.P. Gao). pGL3-IL-6-Luc was kindly provided by Espen Spangenburg. We thank Koichi Hagiwara for the 11-18 cell line. Opinions expressed are solely those of the authors and do not necessarily reflect those of the New York State Department of Health or the State of New York.

Received for publication February 19, 2007, and accepted in revised form September 5, 2007.

Address correspondence to: Jacqueline F. Bromberg, Department of Medicine, Memorial Sloan-Kettering Cancer Center, 1275 York Avenue, New York, New York 10021, USA. Phone: (212) 639-2577; Fax: (646) 422-2231; E-mail: bromberj@mskcc.org.
1. Jett, J.R., and Midthun, D.E. 2004. Screening for lung cancer: current status and future directions: Thomas A. Neff lecture. Chest. 125:158S-162S.

2. Hayes, D.N., et al. 2006. Gene expression profiling reveals reproducible human lung adenocarcinoma subtypes in multiple independent patient cohorts. J. Clin. Oncol. 24:5079-5090.

3. Lynch, T.J., et al. 2004. Activating mutations in the epidermal growth factor receptor underlying responsiveness of non-small-cell lung cancer to gefitinib. N. Engl. J. Med. 350:2129-2139.

4. Paez, J.G., et al. 2004. EGFR mutations in lung cancer: correlation with clinical response to gefitinib therapy. Science. 304:1497-1500.

5. Riely, G.J., Politi, K.A., Miller, V.A., and Pao, W. 2006. Update on epidermal growth factor receptor mutations in non-small cell lung cancer. Clin. Cancer Res. 12:7232-7241.

6. Marshall, J. 2006. Clinical implications of the mechanism of epidermal growth factor receptor inhibitors. Cancer. 107:1207-1218.

7. Pao, W., et al. 2004. EGF receptor gene mutations are common in lung cancers from "never smokers" and are associated with sensitivity of tumors to gefitinib and erlotinib. Proc. Natl. Acad. Sci. U. S. A. 101:13306-13311.
8. Song, L., et al. 2006. Dasatinib (BMS-354825) selectively induces apoptosis in lung cancer cells dependent on epidermal growth factor receptor signaling for survival. Cancer Res. 66:5542-5548.

9. Sordella, R., Bell, D.W., Haber, D.A., and Settleman, J. 2004. Gefitinib-sensitizing EGFR mutations in lung cancer activate anti-apoptotic pathways. Science. 305:1163-1167.

10. Tracy, S., et al. 2004. Gefitinib induces apoptosis in the EGFRL858R non-small-cell lung cancer cell line H3255. Cancer Res. 64:7241-7244.

11. Levy, D.E., and Darnell, J.E., Jr. 2002. Stats: transcriptional control and biological impact. Nat. Rev. Mol. Cell Biol. 3:651-662.

12. Bromberg, J. 2002. Stat proteins and oncogenesis. J. Clin. Invest. 109:1139-1142.

13. Yu, H., and Jove, R. 2004. The STATs of cancer-new molecular targets come of age. Nat. Rev. Cancer. 4:97-105.

14. Haura, E.B., Zheng, Z., Song, L., Cantor, A., and Bepler, G. 2005. Activated epidermal growth factor receptor-Stat-3 signaling promotes tumor survival in vivo in non-small cell lung cancer. Clin. Cancer Res. 11:8288-8294.

15. Mukohara, T., et al. 2003. Expression of epidermal growth factor receptor (EGFR) and downstream- activated peptides in surgically excised non-smallcell lung cancer (NSCLC). Lung Cancer. 41:123-130.

16. Seki, Y., et al. 2004. STAT3 and MAPK in human lung cancer tissues and suppression of oncogenic growth by JAB and dominant negative STAT3. Int. J. Oncol. 24:931-934.

17. Bowman, T., Garcia, R., Turkson, J., and Jove, R. 2000. STATs in oncogenesis. Oncogene. 19:2474-2488.

18. Bromberg, J.F., et al. 1999. Stat3 as an oncogene. Cell. 98:295-303.

19. Haura, E.B., Turkson, J., and Jove, R. 2005. Mechanisms of disease: Insights into the emerging role of signal transducers and activators of transcription in cancer. Nat. Clin. Pract. Oncol. 2:315-324.

20. Ishihara, K., and Hirano, T. 2002. Molecular basis of the cell specificity of cytokine action. Biochim. Biophys. Acta. 1592:281-296.

21. Kishimoto, T. 2005. Interleukin-6: from basic science to medicine -40 years in immunology. Annu. Rev. Immunol. 23:1-21.

22. Yeh, H.H., Lai, W.W., Chen, H.H., Liu, H.S., and Su, W.C. 2006. Autocrine IL-6-induced Stat 3 activation contributes to the pathogenesis of lung adenocarcinoma and malignant pleural effusion. Oncogene. 25:4300-4309.

23. Scheller, J., Ohnesorge, N., and Rose-John, S. 2006. 
Interleukin-6 trans-signalling in chronic inflammation and cancer. Scand. J. Immunol. 63:321-329.

24. Garcia, R., et al. 2001. Constitutive activation of Stat 3 by the Src and JAK tyrosine kinases participates in growth regulation of human breast carcinoma cells. Oncogene. 20:2499-2513.

25. Lee, T.L., Yeh, J., Van Waes, C., and Chen, Z. 2006. Epigenetic modification of SOCS-1 differentially regulates STAT3 activation in response to interleukin- 6 receptor and epidermal growth factor receptor signaling through JAK and/or MEK in head and neck squamous cell carcinomas. Mol. Cancer Ther. 5:8-19.

26. Politi, K., et al. 2006. Lung adenocarcinomas induced in mice by mutant EGF receptors found in human lung cancers respond to a tyrosine kinase inhibitor or to down-regulation of the receptors. Genes Dev. 20:1496-1510.

27. Song, L., Turkson, J., Karras, J.G., Jove, R., and Haura, E.B. 2003. Activation of Stat3 by receptor tyrosine kinases and cytokines regulates survival in human non-small cell carcinoma cells. Oncogene. 22:4150-4165.

28. Alvarez, J.V., Greulich, H., Sellers, W.R., Meyerson, M., and Frank, D.A. 2006. Signal transducer and activator of transcription 3 is required for the oncogenic effects of non-small-cell lung cancerassociated mutations of the epidermal growth factor receptor. Cancer Res. 66:3162-3168.

29. Nagai, Y., et al. 2005. Genetic heterogeneity of the epidermal growth factor receptor in non-small cell lung cancer cell lines revealed by a rapid and sensitive detection system, the peptide nucleic acid-locked nucleic acid PCR clamp. Cancer Res. 65:7276-7282.

30. Pedranzini, L., et al. 2006. Pyridone 6, a panjanus-activated kinase inhibitor, induces growth inhibition of multiple myeloma cells. Cancer Res. 66:9714-9721.

31. Flowers, L.O., Subramaniam, P.S., and Johnson, H.M. 2005. A SOCS-1 peptide mimetic inhibits both constitutive and IL- 6 induced activation of STAT3 in prostate cancer cells. Oncogene. 24:2114-2120.

32. Shouda, T., et al. 2006. Suppression of IL-6 production and proliferation by blocking STAT3 activation in malignant soft tissue tumor cells. Cancer Lett. 231:176-184.

33. Sriuranpong, V., et al. 2003. Epidermal growth factor receptor-independent constitutive activation of STAT3 in head and neck squamous cell carcinoma is mediated by the autocrine/paracrine stimulation of the interleukin 6/gp130 cytokine system. Cancer Res. 63:2948-2956.

34. Sehgal, P.B. 1993. Interlenkin-6. CRC Press. Boca Raton, Florida, USA. 289-307.

35. Xiao, W., et al. 2004. Co-operative functions between nuclear factors NFkappaB and CCAT/enhancerbinding protein-beta (C/EBP-beta) regulate the IL-6 promoter in autocrine human prostate cancer cells.
Prostate. 61:354-370.

36. Haura, E.B., Livingston, S., and Coppola, D. 2006. Autocrine interleukin-6/interleukin-6 receptor stimulation in non-small-cell lung cancer. Clin. Lung Cancer. 7:273-275.

37. Shao, H., Cheng, H.Y., Cook, R.G., and Tweardy, D.J. 2003. Identification and characterization of signal transducer and activator of transcription 3 recruitment sites within the epidermal growth factor receptor. Cancer Res. 63:3923-3930.

38. Zhang, T., Ma, J., and Cao, X. 2003. Grb2 regulates Stat3 activation negatively in epidermal growth factor signalling. Biochem. J. 376:457-464.

39. Sharma, S.V., et al. 2006. A common signaling cascade may underlie "addiction" to the Src, BCR$\mathrm{ABL}$, and EGF receptor oncogenes. Cancer Cell. 10:425-435.

40. Pao, W., et al. 2005. Acquired resistance of lung adenocarcinomas to gefitinib or erlotinib is associated with a second mutation in the EGFR kinase domain. PLoS Med. 2:e73.

41. Leaman, D.W., et al. 1996. Roles of JAKs in activation of STATs and stimulation of c-fos gene expression by epidermal growth factor. Mol. Cell. Biol. 16:369-375.

42. Selander, K.S., et al. 2004. Inhibition of gp130 signaling in breast cancer blocks constitutive activation of Stat 3 and inhibits in vivo malignancy. Cancer Res. 64:6924-6933.

43. Wehbe, H., Henson, R., Meng, F., Mize-Berge, J., and Patel, T. 2006. Interleukin- 6 contributes to growth in cholangiocarcinoma cells by aberrant promoter methylation and gene expression. Cancer Res. 66:10517-10524.

44. Knupfer, H., and Preiss, R. 2006. Significance of interleukin-6 (IL-6) in breast cancer (review). Breast Cancer Res. Treat. 102:129-135.

45. Yanagawa, H., et al. 1995. Serum levels of interleukin 6 in patients with lung cancer. Br. J. Cancer. 71:1095-1098

46. Grant, S.L., et al. 2002. An unexpected biochemical and functional interaction between gp130 and the EGF receptor family in breast cancer cells. Oncogene. 21:460-474.

47. Qiu, Y., Ravi, L., and Kung, H.J. 1998. Requirement of ErbB2 for signalling by interleukin- 6 in prostate carcinoma cells. Nature. 393:83-85.

48. Sato, M., et al. 2006. Multiple oncogenic changes (K-RAS(V12), p53 knockdown, mutant EGFRs, p16 bypass, telomerase) are not sufficient to confer a full malignant phenotype on human bronchial epithelial cells. Cancer Res. 66:2116-2128.

49. Sehgal, P.B. 1992. Regulation of IL6 gene expression. Res. Immunol. 143:724-734.

50. Squarize, C.H., et al. 2006. Molecular cross-talk between the NFkappaB and STAT3 signaling pathways in head and neck squamous cell carcinoma.
Neoplasia. 8:733-746.

51. Park, J.I., et al. 2003. Transforming growth factorbeta 1 activates interleukin- 6 expression in prostate cancer cells through the synergistic collaboration of the Smad2, p38-NF-kappaB, JNK, and Ras signaling pathways. Oncogene. 22:4314-4332.

52. Bancroft, C.C., et al. 2002. Effects of pharmacologic antagonists of epidermal growth factor receptor, PI3K and MEK signal kinases on NF-kappaB and AP-1 activation and IL- 8 and VEGF expression in human head and neck squamous cell carcinoma lines. Int. J. Cancer. 99:538-548.

53. Yang, J., et al. 2007. Unphosphorylated STAT3 accumulates in response to IL- 6 and activates transcription by binding to NFkappaB. Genes Dev. 21:1396-1408.

54. Judd, L.M., et al. 2006. STAT3 activation regulates growth, inflammation, and vascularization in a mouse model of gastric tumorigenesis. Gastroenterology. 131:1073-1085.

55. Soule, H.D., et al. 1990. Isolation and characterization of a spontaneously immortalized human breast epithelial cell line, MCF-10. Cancer Res. 50:6075-6086

56. Thompson, J.E., et al. 2002. Photochemical preparation of a pyridone containing tetracycle: a Jak protein kinase inhibitor. Bioorg. Med. Chem. Lett. 12:1219-1223.

57. Elenbaas, B., et al. 2001. Human breast cancer cells generated by oncogenic transformation of primary mammary epithelial cells. Genes Dev. 15:50-65.

58. Ho, H.H., and Ivashkiv, L.B. 2006. Role of STAT3 in type I interferon responses. Negative regulation of STAT1-dependent inflammatory gene activation. J. Biol. Chem. 281:14111-14118.

59. Morgenstern, J.P., and Land, H. 1990. Advanced mammalian gene transfer: high titre retroviral vectors with multiple drug selection markers and a complementary helper-free packaging cell line. Nucleic Acids Res. 18:3587-3596.

60. Dechow, T.N., et al. 2004. Requirement of matrix metalloproteinase- 9 for the transformation of human mammary epithelial cells by Stat3-C. Proc. Natl. Acad. Sci. U. S. A. 101:10602-10607.

61. Spangenburg, E.E., Brown, D.A., Johnson, M.S., and Moore, R.L. 2006. Exercise increases SOCS-3 expression in rat skeletal muscle: potential relationship to IL-6 expression. J. Physiol. 572:839-848.

62. Rincon, M., et al. 2006. Interleukin-6, multidrug resistance protein-1 expression and response to paclitaxel in women with metastatic breast cancer: results of cancer and leukemia group B trial 159806. Breast Cancer Res. Treat. 100:301-308.

63. Pan, Q., Pao, W., and Ladanyi, M. 2005. Rapid polymerase chain reaction-based detection of epidermal growth factor receptor gene mutations in lung adenocarcinomas. J. Mol. Diagn. 7:396-403. 